\title{
Specificity of Toll-Like Receptor 2 and Dectin-1 Signaling in CNS Macrophages
}

\author{
Deepak Kumar Vijaya Kumar, ${ }^{1}$ William A. Eimer, ${ }^{1}$ and ${ }^{\circledR}$ Sreejith Ramakrishnan ${ }^{2}$ \\ ${ }^{1}$ Genetics and Aging Research Unit, Department of Neurology, Massachusetts General Hospital and Harvard Medical School, Charlestown, Massachusetts \\ 02129, and ${ }^{2}$ Departments of Developmental Biology and Genetics, Stanford University, Stanford, California 94305 \\ Review of Gensel, Wang et al.
}

The first line of innate immune defense in the CNS is mediated by the resident macrophages, that is, microglia. Microglia are the most abundant of all CNS macrophages, which also include meningeal macrophages and macrophages of the choroid plexus and circumventricular organs (Perry and Teeling, 2013). CNS macrophages are activated by primitive ligand-receptor interactions, which initiate a complex downstream cascade of cellular signaling. Macrophages are activated in response to brain or spinal cord injury and are necessary for restoring homeostasis to the CNS. In addition to their reparative functions, CNS macrophages secrete a combination of proinflammatory cytokines and neurotoxins, including TNF- $\alpha$, IL- $1 \beta$, reactive oxygen species, nitric oxide, and glutamate (Stirling et al., 2014). These accumulating proinflammatory cytokines and neurotoxins mount an escalating neuroinflammatory reaction, which, if maintained, results in neuronal loss and neurodegeneration, a hallmark of many CNS pathologies.

Pattern recognition receptors (PRRs) expressed on the surface of microglia and macrophages bind to ligands expressed on

Received Sept. 15, 2015; revised Nov. 3, 2015; accepted Nov. 10, 2015

We thank Dr. Robert D. Moir for providing critical feedback during the preparation of this manuscript.

The authors declare no competing financial interests.

Correspondence should be addressed to Deepak Kumar Vijaya Kumar, 114 16th Street, Charlestown, MA 02129. E-mail: kumar.deepak@mgh.harvard.edu.

DOI:10.1523/JNEUROSCI.3453-15.2015

Copyright $\odot 2015$ the authors $\quad 0270-6474 / 15 / 3516015-03 \$ 15.00 / 0$ bacteria, fungi, and viruses. These ligands are referred to as pathogen-associated molecular patterns (PAMPs). In addition to binding PAMPs, PRRs identify and bind intracellular host molecules that are released from dead and dying tissues after injury or trauma. These host-associated endogenous molecules known as damage-associated molecular patterns (DAMPs) are sometimes collectively referred to as "alarmins" (Bianchi, 2007). Alarmins include molecules like high-mobility group box 1 (HMGB1) proteins, heat-shock proteins, S100 proteins, IL- $1 \alpha$, uric acid, and hepatoma-derived growth factor.

Toll-like receptors (TLRs) and C-type lectin receptors (CLRs) are two of many PRR subtypes that bind specific molecular moieties and trigger macrophagemediated immune activation in the CNS. While TLR2 recognizes bacterial, fungal, and viral PAMPs as well as some DAMPs, dectin-1 has higher specificity for $\beta$ glucans and carbohydrates found on yeast cell walls. In addition, molecular sequences on Mycobacteria cell walls and some host DAMPs also serve as ligands for dectin-1 (Brown, 2006; Kigerl et al., 2009).

A recent study by Gensel, Wang, and colleagues (2015) published in The Journal of Neuroscience demonstrates how selective TLR2 or dectin-1 activation modulates the overall functionality of CNS macrophages. Zymosan (an agonist for both TLR2 and dectin-1), PAM2CSK4 (a selective agonist of TLR2), and TLR2-depleted zymosan (selective for dectin-1) produced varied degrees of CNS injury when microinjected into intact rat spinal cord. Compared with the increased axonal loss and demyelination resulting from dectin-1 selective activation, activation of TLR 2 alone produced minimal effect. Notably, pathology and axonal loss were limited when both TLR2 and dectin-1 were activated (via zymosan), suggesting that coactivation of these receptor subtypes reduced the more damaging effects caused by activation of dectin-1 alone (via TLR2 depleted zymosan) (Gensel, Wang et al., 2015, their Fig. 1). Confocal microscopic examination of markers for axonal injury (neurofilaments and $\beta$-APP) confirmed that axonal injury occurred with dectin-1 alone or dectin-1 plus TLR2 activation, but not with TLR2 alone. In addition, fragmented dystrophic axons were found in tissues activated via dectin-1 alone, whereas TLR2-only activated tissues showed largely unfragmented axon trajectories, even in macrophage-occupied areas (Gensel, Wang et al., 2015, their Fig. 2). These data provide persuasive evidence that macrophages activated by TLR2 are neuroprotective.

Next, Gensel, Wang et al. (2015) used an in vitro assay with bone-marrowderived macrophages to demonstrate that macrophages activated by TLR2-specific agonists secrete neuroprotective factors. Dorsal root ganglion (DRG) neurons isolated from WT C57BL/6 mice were cultured in macrophage-conditioned media 
(MCM) from macrophages stimulated with zymosan, TLR2-depleted zymosan, or PAM2CSK4. MCM from PAM2CSK4 (TLR2 selective)-treated macrophages nurtured DRG neurons and promoted axonal growth. In contrast, MCM from zymosan or TLR2-depleted zymosantreated macrophages caused significant axonal loss and death of DRG neurons (Gensel, Wang et al., 2015, their Fig. 3).

Spinal cord injury (SCI) triggers activation of microglia and macrophages that results in the cells' recruitment to injury sites. Despite a remarkable capacity to repair injured tissue, activated CNS macrophages can also damage host tissues. Macrophages appear to mediate axonal dieback if axons are severed and fail to regenerate (Stirling et al., 2014). The pathways by which macrophages mediate axonal injury and demyelination are only partially understood. To help elucidate possible pathways, Gensel, Wang et al. (2015) tested whether the axonal dieback that follows spinal cord injury could be blocked by selective manipulation of macrophage activity in rats. Two days after SCI, zymosan, TLR2-depleted zymosan, or PAM2CSK4 was injected, and dorsal sensory axons were labeled with fluorescentlabeled dextran. Consistent with previous findings, TLR2 selective activation resulted in significantly reduced axon dieback compared with vehicle injection alone (Gensel, Wang et al., 2015, their Fig. 4). OX42 (a monoclonal antibody against CD11b on macrophages) staining was increased in the lesion site (despite reduced lesion size), suggesting that the attenuation of axonal dieback resulted from enhanced macrophage activity mediated by TLR2 selective activation (Gensel, Wang et al., 2015, their Fig. 5). Furthermore, vimentin, an intermediate filament protein, was significantly increased in tissues injected with TLR2 agonist (PAM2CSK4), and it colocalized with NG2 glial cells. This is notable because previous studies have shown that glial progenitor cells expressing vimentin impair macrophage-mediated axon dieback by providing an axon-stabilizing microenvironment (Busch et al., 2010). Finally, axon dieback in the corticospinal tract was significantly reduced after SCI in dectin-1 knock-out mice compared with wild-type littermates, even though macrophage activation and lesion size were similar in knockout and controls.

The study by Gensel, Wang et al. (2015) is consistent with earlier findings that suggest TLR2-mediated signaling confers protection during injury or trauma. However, important questions remain to be addressed. While Gensel, Wang et al. (2015) demonstrate the neuroprotective properties of TLR2 signaling, additional functional benefits that may co-occur with reduced axonal damage from dectin-1 blockade are left unexplored. Comparing cytokine profiles of macrophages treated with PAM2CSK4 or depleted zymosan may provide useful data. Measuring levels of TNF- $\alpha$ and IL- $1 \beta$, proinflammatory cytokines implicated in CNS pathologies may be particularly informative. Do the beneficial axon regenerating aspects of TLR2 signaling correspond to reduced TNF- $\alpha$ or IL1- $\beta$ secretion? In addition, examining the levels of chondroitin sulfate proteoglycans and glutamate might prove informative, because neurotoxic effects resulting from upregulation of these molecules were found in SCI and trauma (Fitch and Silver, 1997; Liu et al., 1999).

A compromised blood-brain barrier (BBB) has been reported in SCI and trauma. This allows peripheral immune cells to enter the brain, but also provides access to pathogens such as Candida. The primary function of dectin-1 is to initiate an immune response against these pathogens. However, dectin-1-mediated activation of macrophages further increases inflammation, which may make the BBB more permeable and allow further invasion by yeast, further amplifying inflammation. Although dectin-1 is an essential PRR subtype of the CNS, its antifungal role could be doubleedged, with both risks and benefits to the CNS. Cytokines can enhance BBB permeability and alter the immune cell trafficking ability of BBB (Banks et al., 1995; Yang et al., 1999). The findings of Gensel, Wang et al. (2015) demonstrate the intrinsic role that dectin-1 activation may play in exacerbating this cytokine-mediated damage arising with a compromised $\mathrm{BBB}$ in SCI and trauma. However, a measure of the proinflammatory cytokines secreted in response to dectin-1 activation would further strengthen their findings.

The pathophysiology of Alzheimer's disease $(\mathrm{AD})$ is characterized by severe CNS inflammation accompanied by elevated levels of cytokines such as TNF- $\alpha$, IL- $\beta$, IL- 6 , and IFN- $\gamma$ (RubioPerez and Morillas-Ruiz, 2012). Studies have suggested that the BBB is compromised in $\mathrm{AD}$ brains, and two recent studies showed the presence of Candida spp in brains from patients with Alzheimer's disease (Sharma et al., 2012; Pisa et al., 2015a,b). As discussed above, activation of dectin-1 could lead to escalating inflammation in this condition. Gensel, Wang, and colleagues' (2015) work elucidating TLR2 and dectin-1mediated effector functions of CNS macrophages may therefore help explain some of the underlying causes of $\mathrm{AD}$ progression.

CNS complications stemming from the deleterious effects of dectin- 1 selective activation addresses longstanding questions concerning CNS pathology in SCI. Gensel, Wang et al. (2015) have provided valuable data that can be applied to CNS pathologies arising from injury, infection, and degenerative disease. Their findings prompt a key question: can activation of TLRs and CLRs from a minor injury or trauma to the spinal cord or brain result in BBB breakdown, allowing pathogens, toxins, and cytokines to gain access to CNS and start a cascade that compounds pathology from the initial CNS insult?

\section{References}

Banks WA, Kastin AJ, Broadwell RD (1995) Passage of cytokines across the blood-brain barrier. Neuroimmunomodulation 2:241-248. CrossRef Medline

Bianchi ME (2007) DAMPs, PAMPs and alarmins: all we need to know about danger. J Leukoc Biol 81:1-5. Medline

Brown GD (2006) Dectin-1: a signalling nonTLR pattern-recognition receptor. Nat Rev Immunol 6:33-43. CrossRef Medline

Busch SA, Horn KP, Cuascut FX, Hawthorne AL, Bai L, Miller RH, Silver J (2010) Adult $\mathrm{NG} 2+$ cells are permissive to neurite outgrowth and stabilize sensory axons during macrophage-induced axonal dieback after spinal cord injury. J Neurosci 30:255-265. CrossRef Medline

Fitch MT, Silver J (1997) Activated macrophages and the blood-brain barrier: inflammation after CNS injury leads to increases in putative inhibitory molecules. Exp Neurol 148:587-603. CrossRef Medline

Gensel JC, Wang Y, Guan Z, Beckwith KA, Braun KJ, Wei P, McTigue DM, Popovich PG (2015) Toll-like receptors and dectin-1, a C-type lectin receptor, trigger divergent functions in CNS macrophages. J Neurosci 35: 9966-9976. CrossRef Medline

Kigerl KA, Gensel JC, Ankeny DP, Alexander JK, Donnelly DJ, Popovich PG (2009) Identification of two distinct macrophage subsets with divergent effects causing either neurotoxicity or regeneration in the injured mouse spinal cord. J Neurosci 29:13435-13444. CrossRef Medline

Liu D, Xu GY, Pan E, McAdoo DJ (1999) Neurotoxicity of glutamate at the concentration released upon spinal cord injury. Neuroscience 93:1383-1389. CrossRef Medline

Perry VH, Teeling J (2013) Microglia and macrophages of the central nervous system: the contribution of microglia priming and systemic inflammation to chronic neurodegeneration. Semin Immunopathol 35:601-612. CrossRef Medline

Pisa D, Alonso R, Juarranz A, Rábano A, Carrasco L (2015a) Direct visualization of fungal in- 
fection in brains from patients with Alzheimer's disease. J Alzheimers Dis 43:613-624. CrossRef Medline

Pisa D, Alonso R, Rabano A, Rodal I, Carrasco L (2015b) Different brain regions are infected with fungi in Alzheimer's disease. Sci Rep 5:15015. CrossRef Medline

Rubio-Perez JM, Morillas-Ruiz JM (2012) A review: inflammatory process in Alzheimer's disease, role of cytokines. ScientificWorldJournal 2012:756357. CrossRef Medline

Sharma HS, Castellani RJ, Smith MA, Sharma A (2012) The blood-brain barrier in Alzheimer's disease: novel therapeutic targets and nanodrug delivery. Int Rev Neurobiol 102:4790. CrossRef Medline

Stirling DP, Cummins K, Mishra M, Teo W, Yong VW, Stys P (2014) Toll-like receptor 2-med- iated alternative activation of microglia is protective after spinal cord injury. Brain 137: 707-723. CrossRef Medline

Yang GY, Gong C, Qin Z, Liu XH, Lorris Betz A (1999) Tumor necrosis factor alpha expression produces increased blood-brain barrier permeability following temporary focal cerebral ischemia in mice. Mol Brain Res 69:135-143. CrossRef Medline 